\title{
Hydrogen Bonding Structure and Dynamics of Water at the Dimyristoylphosphatidylcholine Lipid Bilayer Surface from a Molecular Dynamics Simulation ${ }^{\dagger}$
}

\author{
Carlos F. Lopez, Steve O. Nielsen, and Michael L. Klein* \\ Center for Molecular Modeling and Department of Chemistry, University of Pennsylvania, \\ 231 South 34th Street, Philadelphia, Pennsylvania 19104-6323
}

\author{
Preston B. Moore \\ Department of Chemistry and Biochemistry, University of the Sciences in Philadelphia, \\ 600 South 43rd Street, Philadelphia, Pennsylvania 19104
}

Received: November 26, 2003; In Final Form: February 16, 2004

\begin{abstract}
An analysis of the structural and dynamical hydrogen bonding interactions at the lipid water interface from a $10 \mathrm{~ns}$ molecular dynamics simulation of a hydrated dimyristoylphosphatidylcholine (DMPC) lipid bilayer is presented. We find that the average number of hydrogen bonds per lipid oxygen atom varies depending on its position within the lipid. Radial distribution functions are reported for water interacting with lipid oxygen, nitrogen, and phosphorus atoms, as well as for lipid-lipid interactions. The extent of inter- and intramolecular lipid-water-lipid hydrogen bond bridges is explored along with charge pair associations among headgroups of different lipid molecules. We also examine the hydrogen bonding dynamics of water at the lipid surface. A picture emerges of a sticky interface where water that is hydrogen bonded to lipid oxygen atoms diffuses slowly. Hydrogen bonds between water and the double bonded lipid oxygen atoms are longer lived than those to single bonded lipid oxygen atoms, and hydrogen bonds between water and the tail lipid oxygen atoms are longer lived than those to headgroup oxygen atoms. The implications of these results for lateral proton transfer at the interface are also discussed.
\end{abstract}

\section{Introduction}

Important biochemical processes such as membrane insertion, proton transport, and drug delivery depend on events that have an initial interaction at the membrane water interface. Lateral diffusion of lipids or membrane bound proteins can also be influenced by different membrane/water interactions.

Proton diffusion at the membrane surface is a topic of current debate. There are no conclusive results on whether proton diffusion on the membrane surface is feasible and energetically more favorable than the alternative desorption and diffusion through solution. ${ }^{1-5}$ The lateral motion of protons from source to sink sites on membranes is of biochemical importance and tightly coupled to the problem of water diffusion on the lipid surface. ${ }^{6}$ The diffusion of protons at interfaces has been seen along pure protein monolayers and is strongly controlled by packing of the surface. ${ }^{7}$ An alternative view postulates that proton diffusion occurs via a hop and turn mechanism, possibly across the bulk. ${ }^{2}$ Experimental studies of water diffusion at hydrophobic interfaces suggest diffusion coefficients for water around $10^{-10}$ to $10^{-11} \mathrm{~cm}^{2} / \mathrm{s}^{8-11}$ compared to the bulk water coefficient of $10^{-5} \mathrm{~cm}^{2} / \mathrm{s}$.

Only a few NMR studies that focus on structural properties such as the number of hydrogen bonds ( $\mathrm{H}$ bonds) per lipid molecule have been reported. ${ }^{12}$ Structural studies on lipid molecules have shown that $10-11$ water molecules interact with

\footnotetext{
† Part of the special issue "Hans C. Andersen Festschrift".

* Corresponding author. E-mail: klein@cmm.chem.upenn.edu. URL: www.cmm.upenn.edu/ klein. Tel: 215-898-1582. Fax: 215-573-6233.

$\doteqdot$ E-mail: moore@cmm.chem.upenn.edu. URL: www.cmm.upenn.edu// $\sim$ moore .
}

the DMPC headgroup. ${ }^{13,14}$ Sum frequency spectroscopy has been employed to evaluate orientation and relaxation properties of water at different interfaces. ${ }^{15-22}$ Properties of water at hydrophobic surfaces have also been reported, showing that water has a preferential orientation at the surface and demonstrating that such interactions affect the penetration of water into the hydrophobic phase. ${ }^{23}$

Computer simulations of water at a phospholipid interface have a long history. The orientation and polarization of water in the interfacial region has been studied for both DLPE and DMPC; a clathrate shell is observed to form around the choline moiety. ${ }^{24-29}$ The hydrogen bonding structure of water and of the lipid headgroups has been analyzed and the effect of employing different electrostatic cutoffs has been assessed. ${ }^{25,28,30}$ Modeling of proton transport between water molecules near a phospholipid/water interface is not possible using conventional MD force fields. Proton transport can be studied using quantum mechanical methods on small systems for short times ${ }^{31}$ or by employing an empirical valence bond model that is incorporated into a conventional MD simulation. ${ }^{32}$

Dynamical calculations of interface interactions have been performed on phosphatidylethanolamine capped lipids but some simplifications, such as electrostatic cutoffs, or short runs were necessary to make the problem tractable. ${ }^{28,33}$ Recent work by Kusumi and co-workers ${ }^{34,35}$ has focused on the $\mathrm{H}$ bonding structure and three-dimensional structure of the lipid water interface with some attention given to dynamical properties of $\mathrm{H}$ bonds at the interface. However, statistics on different lifetimes of these structures are not extensive and a more detailed inspection of such processes seems worthwhile. 
Computer simulations of interfaces can be a valuable tool to gain both qualitative and quantitative information about such systems. The present study complements previous work in giving a molecular level picture of these interactions. We approach this problem with the analysis of the trajectory from a molecular dynamics simulation of DMPC in a fully hydrated lipid bilayer. The focus of the present paper is to characterize the structural and dynamical interactions at the lipid-water interface including $\mathrm{H}$ bonding, adsorption, and transport.

\section{Methods}

Briefly, we have performed molecular dynamics simulations using the parallelized $\mathrm{CM}^{3} \mathrm{D}$ code, ${ }^{37}$ which includes modern calculation techniques such as the REference System Propagator Algorithm (RESPA) and symplectic integrators to reduce the total wall clock CPU time, ${ }^{38-43}$ and the Ewald summation technique ${ }^{44-47}$ for treatment of long-range electrostatic interactions. The AMBER force field was used to describe the lipid molecules. ${ }^{48,49}$ The SPC/E model was used to described the water interactions..$^{50}$

In our previous paper ${ }^{36}$ we reported carrying out NVT and NPT runs at $333 \mathrm{~K}$ before changing to the NVE ensemble, where we reported an average temperature of $333 \mathrm{~K}$. This is not correct: the NVT and NPT runs were performed at $300 \mathrm{~K}$ and $1 \mathrm{~atm}$. The static quantities (e.g., area per lipid) were derived from these simulations. The final configuration (including the velocities) was used to initiate an NVE run, which had an average temperature of $333 \mathrm{~K}$ as a result of the instantaneous configuration that was selected. This is the NVE data reported in the original paper. For the current manuscript, however, we reran the NVE simulation with a different initial configuration (taken from near the end of the NPT run), which averages to $299 \mathrm{~K}$ and $48 \mathrm{~atm}$. A total of 64 lipids and 1792 water molecules are present in the unit cell with dimensions $41.66 \times 44.20 \times$ $68.84 \AA$, giving an area per lipid of $57.6 \AA$.

Due to our choice of state conditions the results of this simulation should correspond to a DMPC lipid bilayer in the liquid crystalline $\left(\mathrm{L}_{\alpha}\right)$ phase. Throughout the paper the atom labels depicted in Figure 1 are employed. These simulations were run at the Pittsburgh Supercomputing Center (PSC) on 32 nodes of the Cray T3E, on 16 nodes of an SGI origin 2000 at NCSA, and on local machines. The total simulation for the $10 \mathrm{~ns}$ trajectory consumed approximately $60000 \mathrm{CPU}$ hours of single processor computer time.

\section{Inter- and Intramolecular Nonbonding Interactions}

A criterion for the measurement of a $\mathrm{H}$ bond needs to be established to quantify the different interactions between the lipid and water molecules. The definition of a $\mathrm{H}$ bond is adopted from previous studies and is defined as follows: a $\mathrm{H}$ bond exists between any two oxygen atoms when the oxygen-oxygen distance is less than $3.25 \AA$, and an angle between this vector and one $\mathrm{O}-\mathrm{H}$ bond is less than $\hat{\mathrm{A}} 35^{\circ} .{ }^{27,34}$ This definition will be used throughout the paper when discussing $\mathrm{H}$ bonds.

A. H Bond Probabilities. To gain a qualitative perspective on the static associations of $\mathrm{H}$ bonds before we calculate any dynamic quantities, we plot time averaged histograms of the probabilities of $\mathrm{H}$ bond formation for each of the lipid oxygen atoms relative to the water solvent atoms. These histograms are reported in Figure 2. In general, ether oxygen atoms (OEX, with $X=1,2, \mathrm{H} 1, \mathrm{H} 2$ ) are less likely to form a $\mathrm{H}$ bond than carboxylic oxygen atoms (ODX, with $X=1,2, \mathrm{H} 1, \mathrm{H} 2)$.

We see that the OE-tail oxygen atoms have the least probability of making $\mathrm{H}$ bonds (Figure $2 \mathrm{a}, \mathrm{b}$ ). The difference in

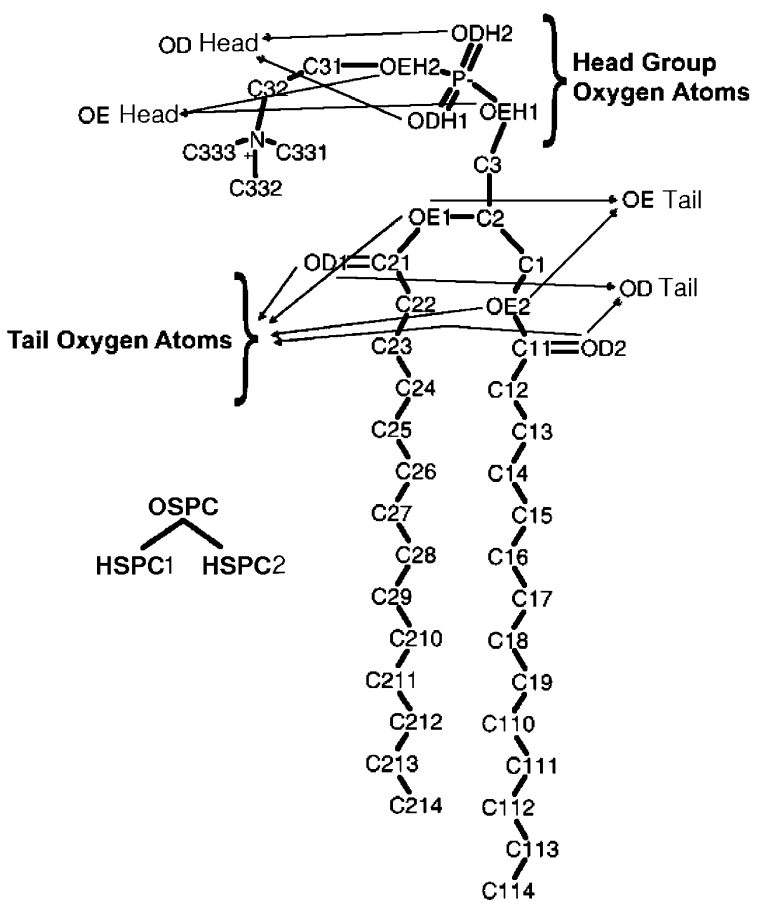

Figure 1. Water and dimyristoylphosphatidylcholine (DMPC) stick drawing with atom names referenced throughout the text.

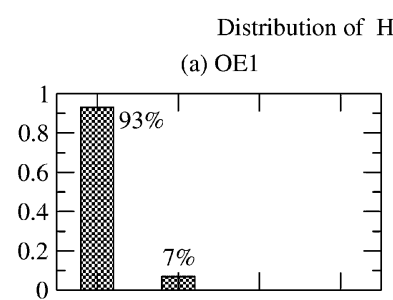

(b) OE2

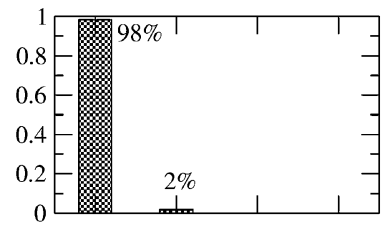

(c) OD1

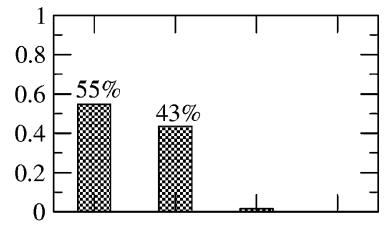

(d) OD2

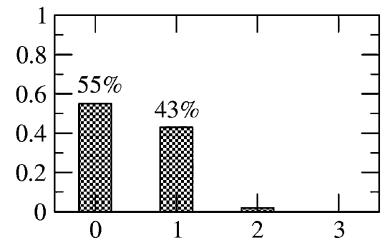

(e) OEH1

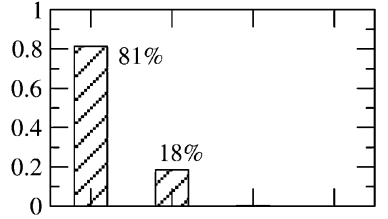

(f) $\mathrm{OEH} 2$

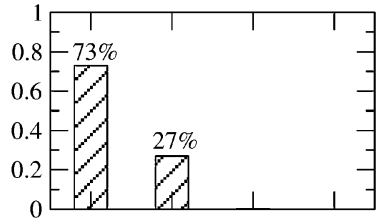

(g) ODH1

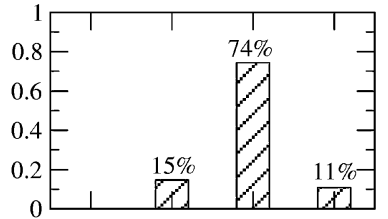

(h) $\mathrm{ODH} 2$

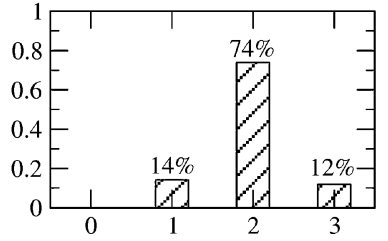

Figure 2. Histograms of the probability of formation of different numbers of hydrogen bonds between lipid and water oxygen atoms. The number on the bottom indicates the number of hydrogen bonds, and the number on the left indicates the probability. The left side is for the tail atoms, and the right side is for the oxygen atoms bonded to the phosphorus. Refer to Figure 1 for the atoms labels.

the probabilities can be attributed to the different environment that is experienced by each individual oxygen atom. The same atom types in the headgroup (OE-head atoms, Figure 2e,f) by comparison have a probability close to $50 \%$ of making one $\mathrm{H}$ 
(a) N-OSPC

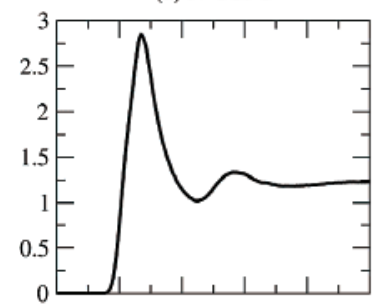

(c) Head Ester O-OSPC

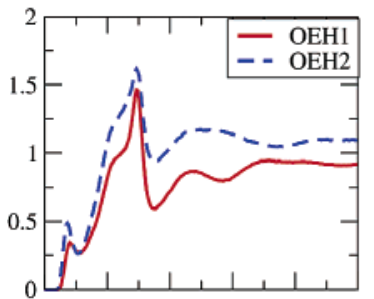

(e) Carbonyl Ester O-OSPC

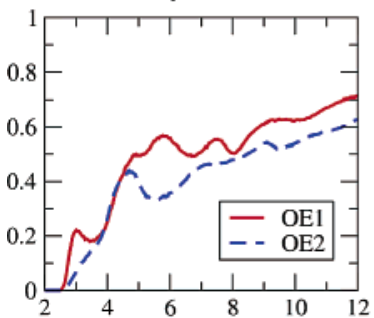

(b) P-OSPC

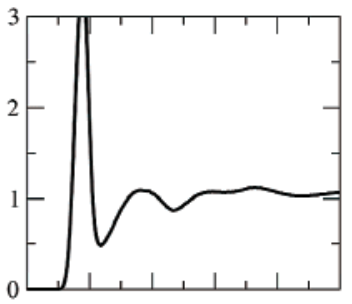

(d) Head non-ester O-OSPC

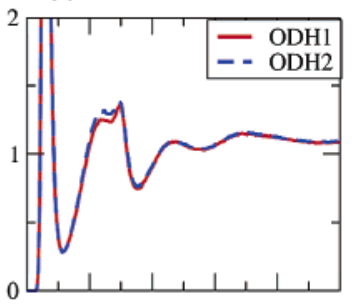

(f) Carbonyl non-ester O-OSPC

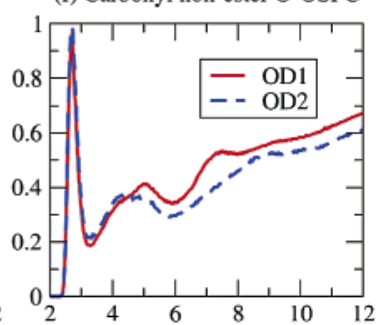

Figure 3. Radial distribution functions for water around different reference atoms: (a) choline nitrogen; (b) phosphate phosphorus; (c), (d) headgroup oxygen atoms; (e), (f) tail oxygen atoms. The radial distribution functions reflect the probability of finding a molecule at a certain distance $(\AA)$ from the central (reference) atom.

bond. This indicates that solvent accessibility is an important factor in $\mathrm{H}$ bonding, because the headgroup will, on average, be in contact with more water molecules than the glycerol moiety.

The probability of $\mathrm{H}$ bond formation is greater for non-ether oxygen atoms. In the case of the OD-tail oxygen atoms (Figure $2 \mathrm{c}, \mathrm{d})$ we find that the probability of formation of one $\mathrm{H}$ bond is $43 \%$ for both OD1 and OD2. The probability of formation of two $\mathrm{H}$ bonds is almost negligible. This can be attributed, at least in part, to the lack of accessibility to water molecules when compared to the OD-head atoms. The $\mathrm{H}$ bond probability changes substantially for the OD-head atoms (Figure 2g,h). For these atoms, the probability of not having a $\mathrm{H}$ bond is negligible, which reflects their extensive contact with the bulk water. These histograms show that, due to the extensive contact of ODH1 and $\mathrm{ODH} 2$ atoms with the solvent, $\mathrm{H}$ bonds will always be present. It is important to note that the contribution of the OEhead atoms, although much less than that of the OD-head atoms, is not negligible and a combination of these two atom types compose the majority of the $\mathrm{H}$ bond interactions at the membrane surface.

B. Hydrogen Bonds and Hydrogen Bridges. We calculate radial distribution functions (RDFs) for the relevant atom-atom interactions, as shown in Figures 3 and 4. All RDFs are normalized by assuming constant uniform density, which is not the case in an inhomogeneous environment, but allows us to integrate the RDFs to obtain coordination numbers in the solvation shells. ${ }^{51}$ We plot RDFs for the nitrogen $(\mathrm{N})$, phosphorus $(\mathrm{P})$, and the lipid oxygen atoms $\mathrm{OD}, \mathrm{OE}, \mathrm{OEH}$, and $\mathrm{ODH}$ because these are the atoms that can form $\mathrm{H}$ bonds with the water oxygen atoms at the interface. Information pertaining to the formation of primary, secondary, or more interaction shells can be extracted from these functions, which yield spatial

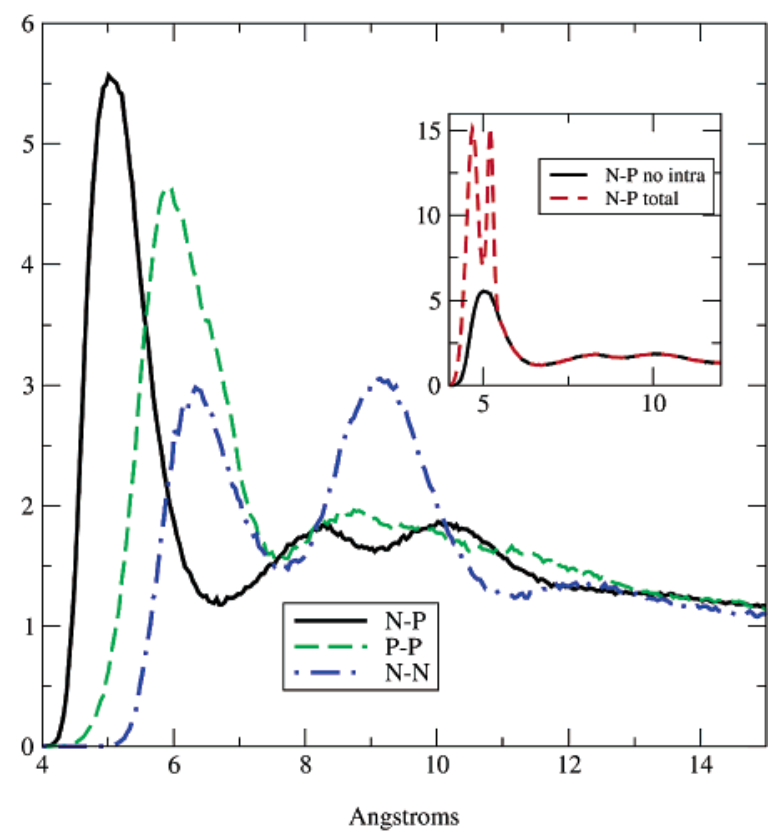

Figure 4. Radial distribution functions for charge pairs involving choline nitrogen and phosphate phosphorus. The inset has the total as well as only the intermolecular contributions. The $\mathrm{P}-\mathrm{P}$ and the $\mathrm{N}-\mathrm{N}$ radial distribution functions are shown as well.

distribution properties around the lipids. ${ }^{52-54}$ Penetration of the water into the bilayer is evident from electron density profiles and has been discussed in our previous study. ${ }^{36}$

In the particular case of the choline nitrogen-water RDF (Figure 3a, N-OSPC) there is a preferential and highly defined hydration shell at the first peak distance and there is evidence for a well-defined second solvation shell. Altough the water is not directly $\mathrm{H}$ bonded to the nitrogen, it surrounds the choline group in well-defined solvation shells. The choline nitrogen has four water molecules at each face of the tetrahedron formed from the methyls attached to the nitrogen, as can be seen in the three-dimensional distribution function (3DDF) of Figure 6. From Figure 6 we can see that the water molecules will preferentially interact with the choline around three sites between the methyl groups.

Similarly, in the case of the phosphorus-water RDF (Figure $3 \mathrm{~b}, \mathrm{P}-\mathrm{OSPC})$ there are several peaks that show strong interaction with the solvent and evidence of a shoulder, which suggests a weakly formed third hydration shell. The phosphate atoms have two water molecules around them on average where the water molecules are $\mathrm{H}$ bonded to the $\mathrm{ODH}$ atoms. Figure 7 depicts the 3DDF of water oxygen (OSPC) about the phosphate group. At this high-density profile, we see water around the double bonded ODH1 and ODH2 atoms in a toroidal motif. This demonstrates that the water is bonded at a preferred angle. In addition, there is a "bridge" around the OEH2 atom, which connects the density distribution that lies around ODH1 and $\mathrm{ODH} 2$. This bridge arises from a third water $\mathrm{H}$ bonding to both OD-bound waters. We can therefore conclude that the phosphate will be solvated by water molecules in contact primarily with the OD-head atoms.

The OE-head atoms RDF shown in Figure $3 c$ exhibit a welldefined solvation structure. The first peak at $3 \AA$ is from direct $\mathrm{H}$ bonding and the next peak with the shoulder is from water $\mathrm{H}$ bonding to the $\mathrm{OD}$ atoms in the phosphate group. The difference in the pattern of the RDFs can be attributed in part to the difference in position between the two atoms. The OEH1 atom is two bonds below the $\mathrm{OEH} 2$ atom, resulting in a different 
(a) O-Head $\mathrm{N}$
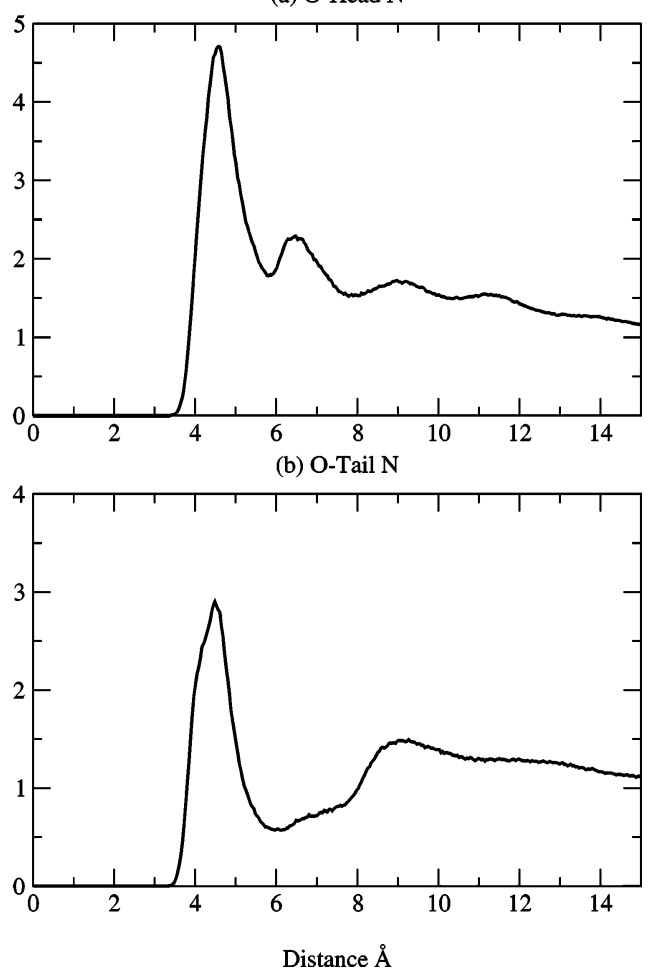

Figure 5. Radial distribution functions for (a) headgroup oxygen atoms (OEH1, OEH2, ODH1, ODH2) and (b) tail oxygen atoms (OE1, OE2, OD1, OD2) around the reference choline nitrogen atom. The radial distribution functions reflect the probability of finding a molecule at a certain distance from the central (reference) atom.

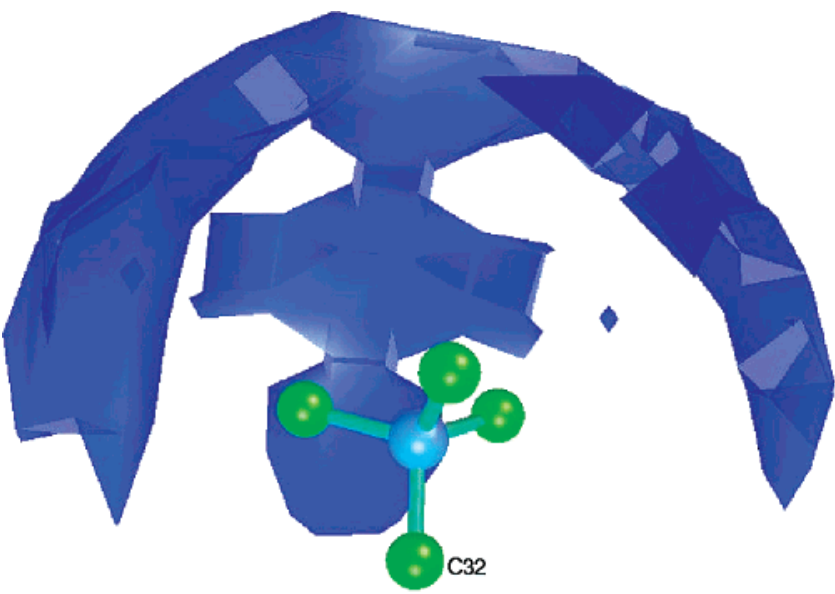

Figure 6. Three-dimensional distribution function of water oxygen atoms around nitrogen choline atoms. $\mathrm{N}$ is the central atom in blue, and carbon atoms are shown in green. The bottom carbon is the C32 carbon, and the other atoms are C331, C332 and C333.

environment, as evidenced by the different probabilities of forming $\mathrm{H}$ bonds (section 3.1), and as shown previously. ${ }^{34}$ This difference would not be noticeable if the whole headgroup was solvated homogeneously. The headgroup, however, lies at an angle of $20^{\circ}$ from the bilayer plane, resulting in an inhomogeneous water environment around the headgroup. ${ }^{52}$ The RDF reflects this different solvent accessible environment for these atoms.

The RDF of the OD-head atoms in DMPC, as shown in Figure $3 d$, is essentially the same for both ODH1 and ODH2, reflecting the symmetry of the molecule. The OD-head atoms are bonded directly to the $\mathrm{P}$ atom and have a similar orientation at the interface. There are two clear hydration shells, and there

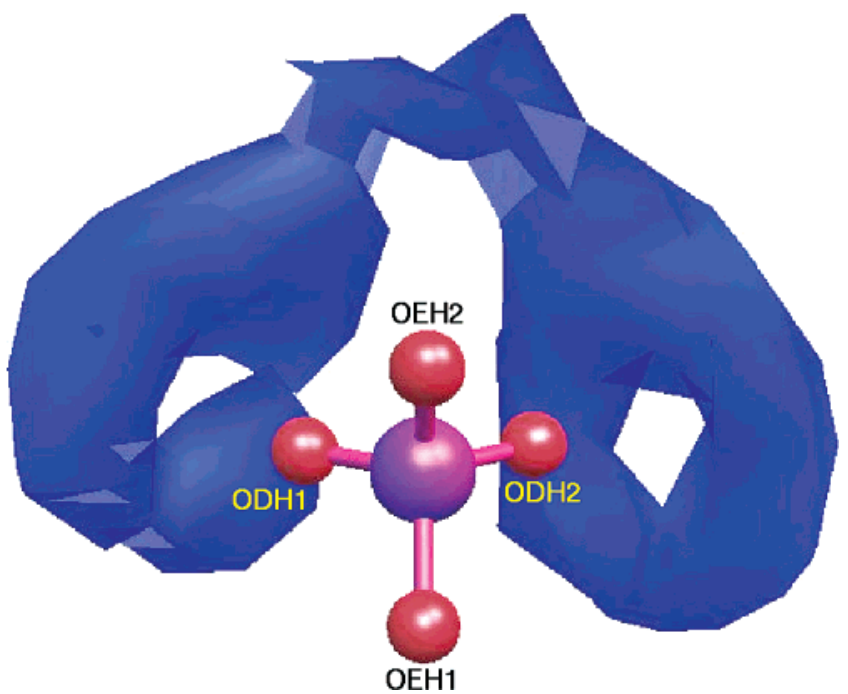

Figure 7. Three-dimensional distribution of water oxygen atoms around the phosphate phosphorus atom. $\mathrm{P}$ is the central atom in blue, and oxygens are shown in red. The bottom oxygen is OEH1, and the top is OEH2. ODH1 and ODH2 are on the left and right, respectively.

is evidence for a third and fourth hydration shell, which is indicative of the accessibility of these atoms to the solvent. Within the second peak there are two lobes: one at $5 \AA$ from the second hydration shell of water in the bulk, and a closer lobe at $4 \AA$ from a water bridge. This water bridge is seen in Figure 7 where the bridging water is $\mathrm{H}$ bonded to two water molecules which are in turn bonded to ODH atoms.

For the OE-tail atoms an effect similar to that seen in the OE-head (Figure 3c) atoms is observed, as shown in Figure 3e. The difference in the local solvation environment results in variations between RDFs. For example, the first small peak at $2.7 \AA$ is from direct $\mathrm{H}$ bonding, and there is a clear peak in OE1 where there is only a shoulder for the OE2 atoms. The density at $5 \AA$ is from water $\mathrm{H}$ bonding to the OD tail atoms and preferential sites on the phosphate headgroup. The OE-tail to water oxygen RDF (Figure 3e) shows that OE2 is slightly deeper within the lipid bilayer than OE1. The patterns of both RDFs are similar beyond a radial distance of $8 \AA$, indicating the presence of bulk liquid at this distance.

For the OD-tail atoms a well-defined first and second solvation shell is observed (Figure $3 \mathrm{f}$ ). The integrated area under the OD1 and OD2 curves and the broader features of the OD2 RDF again indicate that OD2 is more buried than OD1. The interactions of different oxygen atoms with water indicate that $\mathrm{H}$ bonding provides an important contribution to the properties of the lipid-water interface.

There is on average one intermolecular HBB per lipid molecule and one intramolecular HBB per 10 lipid molecule. This means that most molecules will make a $\mathrm{H}$ bridge perhaps in addition to existing $\mathrm{H}$ bonds and $\mathrm{CP}$ interactions. The presence of these interactions throughout the simulation underline their contributions to the structural properties of the membrane surface.

C. Charge Pair Associations. It has been postulated that some type of association exists between headgroups of different lipids. ${ }^{27,55}$ This issue has been addressed recently, ${ }^{35}$ but no conclusive interpretation regarding the formation of these associations and their contributions to the stability of the headgroup environment has been formulated. To explore this phenomenon, we look at the so-called "salt pairs" or "salt bridges" 56 between charged atoms. The choline nitrogen, the phosphorus atom, and the phosphate oxygens are all charged 


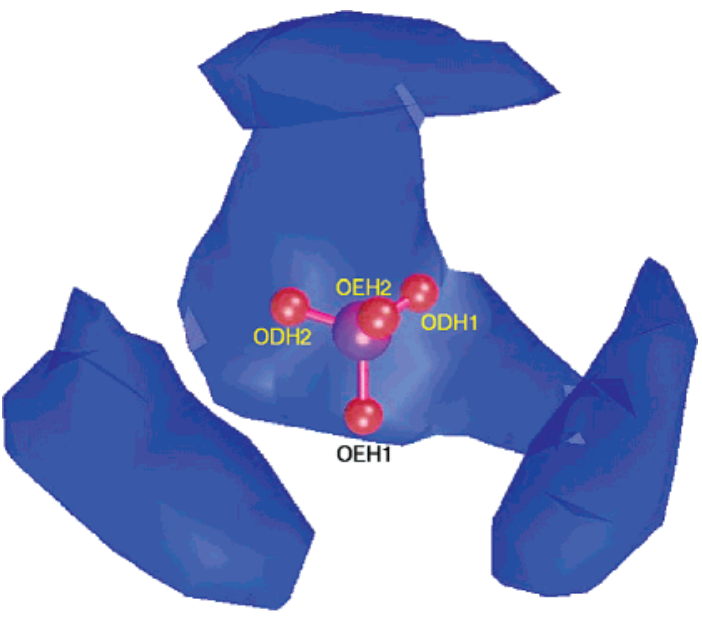

Figure 8. Three-dimensional distribution of choline nitrogen atoms around the phosphate phosphorus atom. $\mathrm{P}$ is the central atom in blue, and oxygens are shown in red. The bottom oxygen is OEH1, and the top is OEH2. ODH1 and ODH2 are on the left and right, respectively.

species. Given the mobility of the headgroup (shown in our previous paper ${ }^{36}$ ), we wish to establish whether such associations between the headgroup oxygens and the choline nitrogen exist, and furthermore whether associations between the carbonyl oxygens and the headgroup can exist as well.

We plot the choline nitrogen-phosphorus (NP), phosphorusphosphorus (PP), and nitrogen-nitrogen (NN) RDFs, as shown in Figure 4. The inclusion of intramolecular interactions in the calculation of the NP RDF is shown in the inset. The two sharp peaks that arise in the inset correspond to the most stable torsional conformations looking along the $\mathrm{C} 31-\mathrm{C} 32$ bond in the sequence $\mathrm{P}-\mathrm{OEH} 2-\mathrm{C} 31-\mathrm{C} 32-\mathrm{N}$ (refer to Figure 1 for the atom labels). Removing the intramolecular contributions to the RDF results in the solid line in the graph, which has a definite interaction shell with a first minimum at $6.7 \AA$ and a peak at $5 \AA$. In addition, there are two more shallow peaks that suggest long range order. The PP RDF shows a similar structure in the first maximum and minimum, again suggesting that there is a preferential association shell indicated by the peaks in the RDF. The NN RDF confirms ordering in the headgroup interaction. The NN and PP RDFs both have a peak at about 6 $\AA$ formed from head-head dipoles. The NN second peak at 9 $\AA$ and the two diffuse peaks of the PP at 9 and $10 \AA$ are due to side by side interactions of the headgroup dipoles.

Figure 8 depicts the spatial distribution of the $\mathrm{N}$ atom about the $\mathrm{P}$ atom using the ideal positions of $\mathrm{OEH} 1$ and $\mathrm{OEH} 2$ as a reference point to define the coordinate system. In this figure we see that there are three preferential sites where the $\mathrm{N}$ can interact with the P. These sites lie between the ODH1, ODH2, and $\mathrm{OEH} 2$ atoms. There are only a handful of preferred ways that these two atoms will interact in a spatially ordered fashion. A complementary view of this interaction is shown in Figure 9 where the $\mathrm{P}$ spatial distribution is shown about the $\mathrm{N}$ with the methyl groups used to define the coordinate system. These density plots suggest not a simple aligned 2D lattice of dipoles, but aligned dipoles that are puckered where the preferred interactions do not lie in a plane.

From the previous discussion we can define two types of interactions for charge pairs. The first is when an oxygen atom and a nitrogen atom are less than $5.8 \AA$ apart (this distance corresponds to the first minimum in the RDF; see Figure 5). The second is when a nitrogen and a phosphorus atom are less than $6.7 \AA$ apart.

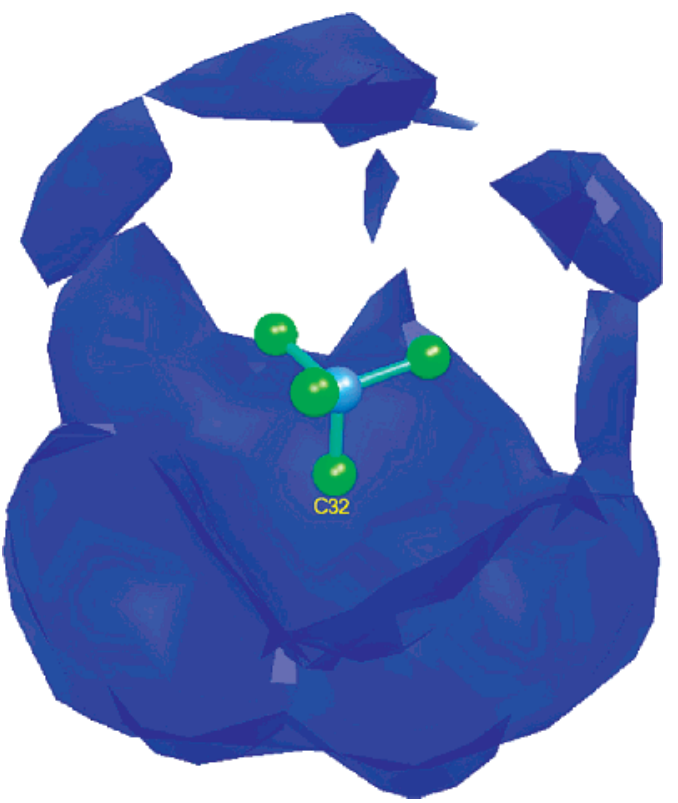

Figure 9. Three-dimensional distribution of phosphorus atoms around the choline nitrogen atom. $\mathrm{N}$ is the central atom in blue, and carbon atoms are shown in green. The bottom carbon is the $\mathrm{C} 32$ carbon.

The combination of $\mathrm{H}$ bond, $\mathrm{HBB}$, and $\mathrm{CP}$ interactions forms a network at the lipid-water interface that is an important factor for the stability, mobility, and dynamics of the interface.

\section{Dynamics of Hydrogen Bonds}

In this section we discuss the analysis methods employed to study hydrogen bonding at the interface, followed by the results obtained.

A. Analysis. To characterize the lifetime of the structures in question, we appeal to the microscopic theory of chemical reaction rates due to Yamamoto. ${ }^{57}$ If we picture the formation and breakup of the structures in question as a simple interconversion process $\mathrm{A} \rightleftharpoons \mathrm{B}$, then the rate constant $k=k_{\text {forward }}$ $+k_{\text {reverse }}$ coming from the corresponding phenomenological mass action kinetic equation is given by

$$
k=\frac{\left\langle j(0) \chi\left(t^{*}\right)\right\rangle}{\left\langle\chi^{2}(0)\right\rangle}
$$

In this expression $\chi$ is the progress variable for the conversion process and $j$ is its reactive flux. The applicability of expression (1) relies on there being a separation of time scales: there should be a time $t^{*}$ such that $\tau_{\text {micro }} \ll t^{*} \ll \tau_{\text {chem }}$, where $\tau_{\text {micro }}$ characterizes the microscopic relaxation time of the system and $\tau_{\text {chem }}=k^{-1}$ is the chemical relaxation time. Replacing $t^{*}$ with $t$ in expression (1) allows us to relate this expression to the correlation function

$$
c(t)=\frac{\langle\chi(0) \chi(t)\rangle}{\left\langle\chi^{2}(0)\right\rangle}
$$

by $k(t)=-\mathrm{d} c / \mathrm{d} t .^{58-60}$ The rate constant $k=k\left(t^{*}\right)$ is obtained from the plateau region in $k(t)$. The infinite time value $k(t=$ $\infty$ ) is not of interest because it is zero, although at long times $k(t)$ has the form $k(t) \approx k^{*} \exp \left(-k^{*} t\right)$, which can also be used to extract the value of $k^{*}$.

The phenomenological rate law breaks down if the chemical relaxation time is not well separated from the microscopic relaxation times in the system. ${ }^{61}$ In this case $k(t)$ does not exhibit a plateau region and the process is not described by a simple 


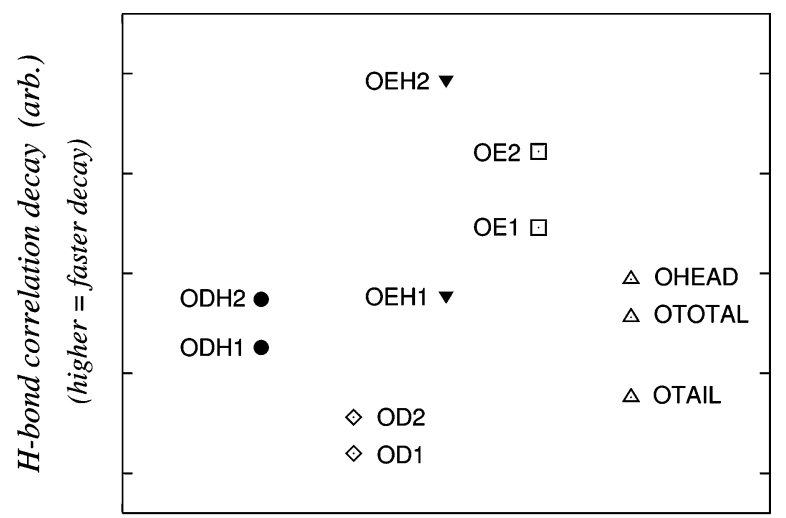

Figure 10. Measure of the decay of the correlation functions (see eq 2 ) for hydrogen bonding between water molecules and lipid oxygen atoms. The eight individual lipid oxygen atoms are shown along with the correlation function for water hydrogen bonded to any headgroup oxygen atom, any tail oxygen atom, and any lipid oxygen atom, respectively. The double bonded oxygen correlation functions have a slower decay than the single bonded ones. In addition, the tail oxygen correlation functions decay slower than the headgroup ones. The values used to characterize the decay are the negative of the integral of the semilog correlation function up to $2 \mathrm{~ns}$. The same ordering is acheived by using the negative of the value of the semilog correlation function at 2 ns.

mass action rate law. Nonetheless, the correlation function $c(t)$ is still of value because it characterizes the nature of the process. By choosing the progress variable $\chi$ to be 1 when a particular hydrogen bond exists and zero when it is broken, the correlation function $c(t)$ is equal to the probability that a particular structure exists given that it was intact at time zero. Hence $c(t)$ reflects the distribution of lifetimes for the structures of interest.

This formalism frees us from the need to use an additional distance or time parameter in deciding whether a given structure has re-formed. ${ }^{34,62}$ The following section uses specific choices of $\chi$ to study dynamical processes at the lipid water interface.

B. Hydrogen Bond Dynamics. To study hydrogen bond dynamics, we define the progress variable $\chi$ as follows. $\chi$ is given a value of 1 if a water oxygen atom and a lipid oxygen atom are separated by less than $3.25 \AA$ and an angle between this vector and one $\mathrm{O}-\mathrm{H}$ vector is less than $35^{\circ}$. The correlation function obtained from $\chi$ gives the probability that a particular water-lipid $\mathrm{H}$ bond is still intact after an interval $t$. The generalized rate coefficient $k(t)$ corresponding to this choice of $\chi$ displays neither a plateau region nor an exponential tail (see Figure 11). This is in accordance with the studies of Luzar and Chandler ${ }^{58-60}$ on $\mathrm{H}$ bond kinetics in bulk water (see Figure 1 of ref 58), in which it is suggested that the time scale separation breakdown is a result of diffusion. We do not attempt to determine the cause of the breakdown for the lipid-water $\mathrm{H}$ bond kinetics, but we note that the functional forms in semilog and $\log -\log$ format (see Figure 11) are strikingly similar to the work in ref 58. Although we cannot extract decay coefficients from the correlation functions, we can nonetheless compare how quickly they decay in a heuristic manner. We characterize the decay (see Figure 10) by the negative of the integral of the semilog correlation function (see Figure 11) up to $2 \mathrm{~ns}$. The same ordering is acheived by using the negative of the value of the semilog correlation function at 2 ns. The ordering in Figure 10 displays the following trends.

Hydrogen bonds between water and the double bonded lipid oxygen atoms (OD1, OD2, ODH1, and ODH2) are longer lived than those to single bonded lipid oxygen atoms (OE1, OE2, $\mathrm{OEH} 1$, and $\mathrm{OEH} 2$ ), and hydrogen bonds between water and the tail lipid oxygen atoms (OE1, OE2, OD1, OD2) are longer

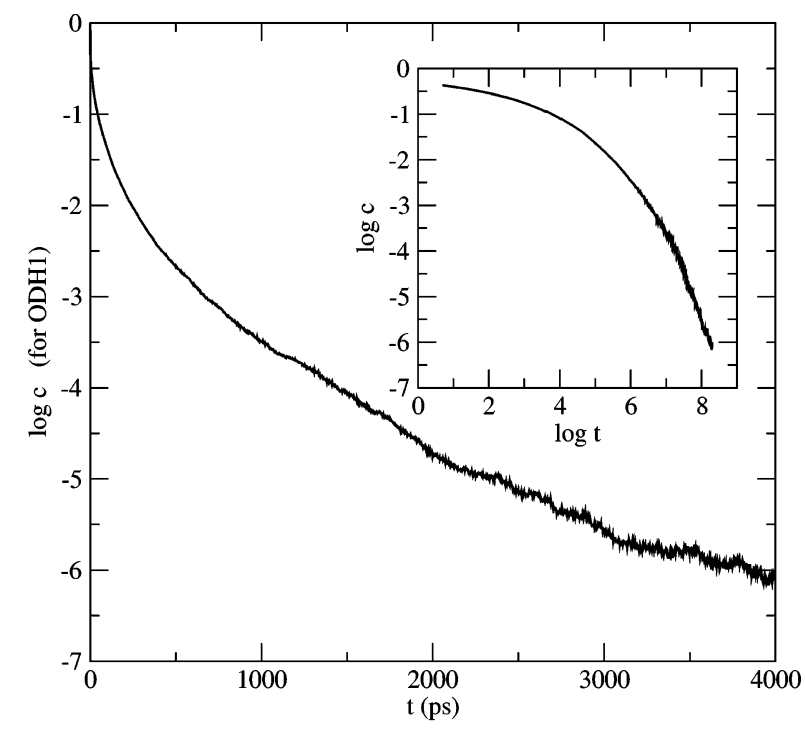

Figure 11. Representative correlation function [see eq 2] in semilog and $\log -\log$ form. The functional form should be compared to Figure 1 of ref 58. See the text for discussion.

lived than those to headgroup oxygen atoms (OEH1, OEH2, ODH1, ODH2). The eight individual lipid oxygen atom correlation functions are supplemented by three correlation functions shown on the right-hand side of Figure 10: namely, with $\chi$ given a value of 1 if a hydrogen bond is formed between a water molecule and any lipid headgroup oxygen atom (labeled OHEAD), any lipid tail oxygen atom (labeled OTAIL), and any lipid oxygen atom (labeled OTOTAL). The most deeply buried lipid oxygen atoms (OD1 and OD2) are double bonded and form very long-lived hydrogen bonds to water molecules. This long lifetime is presumably due to both the strong effect of the double bond and to the lack of competition for hydrogen bonds at this depth below the interface. The strong effect of the double bond on lifetimes is shown by the double bonded headgroup oxygen atoms (ODH1 and ODH2); water is in much greater competition to form hydrogen bonds to other atoms, but it still forms a longlived hydrogen bond in this situation. The competition for hydrogen bonding with water is most clearly shown by the fast decay associated with the $\mathrm{OEH} 2$ species (see Figure 1); this atom is completely exposed to the water subphase and its single bonded nature makes it a poor competitor.

C. Dynamics of Water at the Interface. The last dynamical property that we investigate is the diffusion of water at the interface. The three-dimensional mean square displacement of water molecules hydrogen bonded to the lipid oxygen atoms is measured (see Figure 12) for water associated with the headgroup region (atoms OEH1, OEH2, ODH1, ODH2), the tail region (atoms OE1, OE2, OD1, OD2), and all lipid oxygen atoms. The diffusion coefficients are $1.9 \times 10^{-7} \mathrm{~cm}^{2} \mathrm{~s}^{-1}$ for the water molecules associated with the headgroup oxygens, $2.3 \times 10^{-7} \mathrm{~cm}^{2} \mathrm{~s}^{-1}$ for the water molecules associated with the tail oxygens, and $2.0 \times 10^{-7} \mathrm{~cm}^{2} \mathrm{~s}^{-1}$ for the water molecules associated with all lipid oxygen atoms. These values are significantly slower than the self-diffusion of pure SPC and SPC/E water, which is roughly $D=3.85 \times 10^{-5} \mathrm{~cm}^{2} \mathrm{~s}^{-1}$ and $D=2.5 \times 10^{-5} \mathrm{~cm}^{2} \mathrm{~s}^{-1}$, respectively, at $298 \mathrm{~K},{ }^{63}$ but are the same order of magnitude as the diffusion of the lipids. The lipid oxygen atoms have a three-dimensional diffusion coefficient of roughly $1.7 \times 10^{-7} \mathrm{~cm}^{2} \mathrm{~s}^{-1}$, which is in agreement with the attached waters. The dynamical results suggest that water molecules can reside in a "trapped" state at the surface, which makes proton transport via a Grotthus type mechanism viable. 


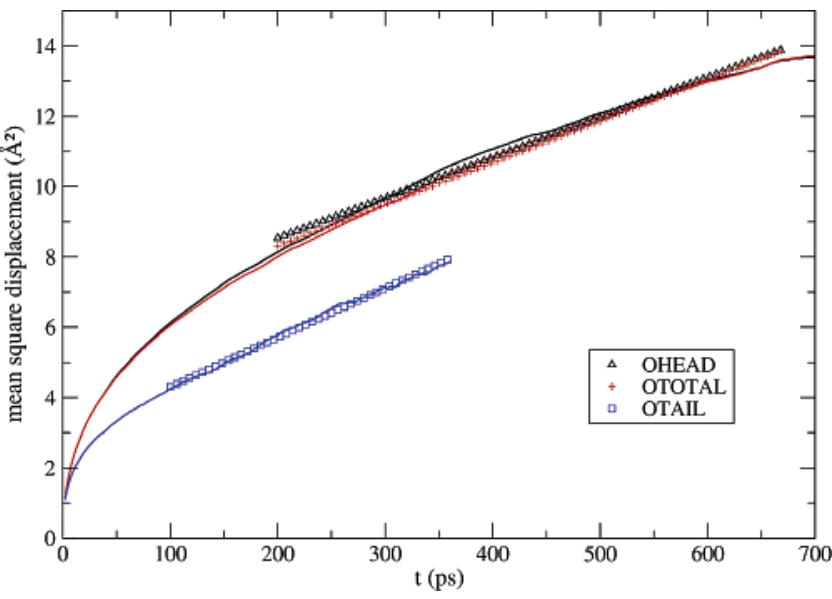

Figure 12. Mean square displacement of water molecules hydrogen bonded to the lipid oxygen atoms. Data are shown for water associated with the headgroup region (atoms OEH1, OEH2, ODH1, ODH2), the tail region (atoms OE1, OE2, OD1, OD2), and any lipid oxygen atom. The three-dimensional diffusion constants are $1.91 \times 10^{-7} \mathrm{~cm}^{2} \mathrm{~s}^{-1}$ for the water molecules associated with the headgroup oxygens, $2.34 \times$ $10^{-7} \mathrm{~cm}^{2} \mathrm{~s}^{-1}$ for the water molecules associated with the tail oxygens, and $1.97 \times 10^{-7} \mathrm{~cm}^{2} \mathrm{~s}^{-1}$ for the water molecules associated with any lipid oxygen atom. The data used to estimate the diffusion constants is shown with symbols.

\section{Conclusion}

We have studied the structural and dynamical hydrogen bonding features at the lipid water interface from a $10 \mathrm{~ns}$ hydrated DMPC simulation. The waters involved in such bonds can participate in lipid-water-lipid bridges; in addition, direct charge pair interactions can form between the headgroups of different lipid molecules. The combination of these three interactions leads to a hydrogen bond/charge pair network at the interface. Most of the structural results are in close agreement with those reported previously. ${ }^{34,35,64}$

We find that the hydrogen bonding kinetics does not obey a phenomenological mass action rate law. This finding is in accordance with the results in pure water reported by Luzar and Chandler, ${ }^{58}$ in which they attribute the breakdown to a coupling between hydrogen bonding and diffusion. It is plausible that the same effect occurs in the present situation, because the hydrogen bonding lifetimes and the diffusion constants are both significantly slower than in pure water. Further work is needed to confirm this interpretation.

Acknowledgment. This work was supported by NIH grant GM40712. We thank the support that NCSA and PSC have provided through their supercomputing time allocations under the NPACI program.

\section{References and Notes} 2602.

(1) Slevin, C. J.; Unwin, P. R. J. Am. Chem. Soc. 2000, 122, 2597-

(2) Prats, M.; Tocanne, J. F.; Teissie, J. Eur. J. Biochem. 1987, 162, $379-385$.

(3) Teissie, J.; Prats, M.; LeMassu, A.; Stewart, L. C.; Kates, M. Biochemistry 1990, 29, 59-65.

(4) Scherrer, P. Nature 1995, 374, 222.

(5) Teissie, J.; Scherrer, P. Nature 1996, 379, 305.

(6) Gennis, R. B. Biomembranes: Molecular Structure and Function; Springer-Verlag: New York, : 1989.

(7) Gabriel, B.; Teissie, J. Proc. Natl. Acad. Sci. U.S.A. 1996, 93, $14521-14525$

(8) Tanzawa, K.; Hirota, N.; Terazima, M. Chem. Phys. Lett. 1997, $274,159-164$. 10309
(10) Girard, K. P.; Quinn, J. A.; Vandelick, T. K. J. Colloid Interface Sci. 1999, 217, 146-153.

(11) Ciampi, E.; Goerke, U.; Keddie, J.; MacDonald, P. Langmuir 2000, $16,1057-1065$

(12) Nagle, J. F. Biophys. J. 1993, 64, 1476-1481.

(13) Westlund, P. J. Phys. Chem. B 2000, 104, 6059-6064.

(14) Faure, C.; Bonakdar, L.; Dufourc, E. J. FEBS Lett. 1997, 405, $263-$ 266.

(15) Miranda, P. B.; Shen, Y. R. J. Phys. Chem. B 1999, 103, 32923307.

(16) Gragson, D. E.; Richmond, G. L. J. Phys. Chem. B 1998, 102, $569-576$.

(17) Gragson, D. E.; Richmond, G. L. J. Phys. Chem. B 1998, 102, 3847-3861.

(18) Smiley, B. L.; Richmond, G. L. Biopolymers 2000, 57, 117-125.

(19) Richmond, G. L. Annu. Rev. Phys. Chem. 2001, 52, 357-389.

(20) Walker, R. A.; Gragson, D. E.; Richmond, G. L. Colloids Surf. 1999, 154, 175-185.

(21) Benderskii, A. V.; Eisenthal, K. B. J. Phys. Chem. B 2000, 104, $11723-11728$.

(22) Rossky, P. J. Nature 2001, 410, 645, 647-648.

(23) Scatena, L. F.; Brown, M. G.; Richmond, G. L. Science 2001, 292, 908-912.

(24) Charifson, P. S.; Hiskey, R. G.; Pedersen, L. G. J. Comput. Chem. 1990, 11, 1181-1186.

(25) Zhou, F.; Schulten, K. J. Phys. Chem. 1995, 99, 2194-2207.

(26) Berkowitz, M. L.; Raghavan, K. Langmuir 1991, 7, 1042-1044.

(27) Raghavan, K.; Reddy, M. R.; Berkowitz, M. L. Langmuir 1992, 8, 233-240.

(28) Alper, H. E.; Bassolino-Klimas, D.; Stouch, T. R. J. Chem. Phys. 1993, 99, 5547-5559.

(29) Damodaran, K. V.; Merz, K. M. M., Jr. Biophys. J. 1994, 66, 10761087.

(30) Pandit, S. A.; Bostick, D.; Berkowitz, M. L. Biophys. J. 2003, 85, $3120-3131$

(31) Tuckerman, M. E.; Marx, D.; Klein, M. L.; Parrinello, M. Science 1997, 275, 817-820.

(32) Smondyrev, A. M.; Voth, G. A. Biophys. J. 2002, 82, 1460-1468.

(33) Damodaran, K. V.; Merz, K. M. M., Jr.; Gaber, B. P. Biochemistry 1992, 31, 7656-7664.

(34) Pasenkiewicz-Gierula, M.; Takaoka, Y.; Miyagawa, H.; Kitamura, K.; Kusumi, A. J. Phys. Chem. A 1997, 101, 3677-3691.

(35) Pasenkiewicz-Gierula, M.; Takaoka, Y.; Miyagawa, H.; Kitamura, K.; Kusumi, A. Biophys. J. 1999, 76, 1228-1240.

(36) Moore, P. B.; Lopez, C. F.; Klein, M. L. Biophys. J. 2002, 81, 2484-2494

(37) Moore, P. B.; Klein, M. L. Implementation of a General Integration for Extended System Molcular Dynamics. Technical Report, University of Pennsylvania, 1997; http://www.cmm.upenn.edu/ moore/code/code.html

(38) Humphreys, D. D.; Friesner, R. A.; Berne, B. J. J. Chem. Phys 1994, 98, 6885 .

(39) Martyna, G. J.; Tuckerman, M. E.; Tobias, D. J.; Klein, M. L. Mol. Phys. 1996, 87, 1117-1157.

(40) Cheng, A.; Merz, K. M. M., Jr. J. Phys. Chem. B. 1999, 103, 53965405

(41) Tuckerman, M. J.; Berne, B. J.; Martyna, G. J. J. Chem. Phys. 1992 97, 1990-2001.

(42) Evans, D.; Holian, B. J. Chem. Phys. 1985, 83, 4069-4074

(43) Tobias, D. J.; Martyna, G. J.; Klein, M. L. J. Chem. Phys. 1993 97, 12959.

(44) Brown, D.; Neyertz, S. Mol. Phys. 1995, 84, 577.

(45) Nymand, T. M.; Linse, P. J. Chem. Phys. 2000, 112, 6151-6160.

(46) Darden, T.; York, D.; Pedersen, L. J. Chem. Phys. 1993, 98, 1008910092

(47) Essmann, U.; Perera, L.; Berkowitz, M. L.; Darden, T.; Lee, H.; Pedersen, L. J. Chem. Phys. 1995, 103, 8577.

(48) Cornell, W. D.; Cieplak, P.; Bayly, C. I.; Gould, I. R.; Merz, K M.; Ferguson, D. M.; Spellmeyer, D. C.; Fox, T.; Caldwell, J. W.; Kollman, P. A. J. Am. Chem. Soc. 1995, 117, 5179-5197.

(49) Tieleman, D. P.; Marrink, S. J.; Berendsen, H. J. C. Biochim Biophys. Acta 1997, 1331, 235-270.

(50) Berendsen, H. J. C.; Grigera, J. R.; Straatsma, T. P. J. Phys. Chem. 1987, 91, 6269-6271.

(51) Allen, M. P.; Tildesley, D. J. Computer Simulations of Liquids; Oxford University Press: Oxford, U.K., 1987.

(52) Husslein, T.; Newns, D. M.; Pattnaik, P. C.; Zhong, Q.; Moore, P. B.; Klein, M. L. J. Chem. Phys. 1998, 109, 2826-2832.

(53) Koubi, L.; Tarek, M.; Klein, M. L.; Scharf, D. Biophys. J. 2000, $78,800-811$ 2156. 
(55) Pastor, R. W.; Feller, S. E. Time Scales of Lipid Dynamics and Molecular Dynamics. In Biological Membranes; K. Merz, J.; Roux, B., Eds.; Birkhäuser: Boston, 1996.

(56) Stryer, L. Biochemistry, 4th ed.; W. H. Freeman and Co.: New York, 1995

(57) Yamamoto, T. J. Chem. Phys. 1960, 33, 281.

(58) Luzar, A.; Chandler, D. Nature 1996, 379, 55-57.

(59) Luzar, A.; Chandler, D. Phys. Rev. Lett. 1996, 76, 928-931.
(60) Luzar, A. Chem. Phys. 2000, 258, 267-276.

(61) Wu, X.-G.; Kapral, R. J. Chem. Phys. 1989, 91, 5528-5543.

(62) Impey, R. W.; Madden, P. A.; MacDonald, I. R. J. Phys. Chem. 1983, $87,5071-5083$.

(63) Mahoney, M. W.; Jorgensen, W. L. J. Chem. Phys. 2001, 114, $363-$ 366.

(64) Takaoka, Y.; Pasenkiewicz-Gierula, M.; Miyagawa, H.; Kitamura, K.; Tamura, Y.; Kusumi, A. Biophys. J. 2000, 79, 3118-3138. 